\title{
Análise dos registros de enfermagem sobre dor e analgesia em doentes hospitalizados*
}

\author{
ANALYSE NURSING RECORDS ON PAIN AND ANALGESIA OF PATIENTS HOSPITALIZED \\ ANÁLISE DEL REGISTROS DE ENFERMERÍA SOBRE EL DOLOR Y ANALGESIA \\ EN PACIENTES INTERNADOS
}

Yara Boaventura da Silva1, Cibele Andrucioli de Mattos Pimenta²

\author{
RESUMO \\ Estudo que analisou os \\ registros de enfermagem \\ sobre dor e analgesia em \\ doentes internados em um \\ hospital oncológico, de \\ outubro a novembro de 1999 , \\ e os comparou ao relato dos \\ doentes. Entrevistou-se 38 \\ doentes com queixa dolorosa. \\ $O$ registro de enfermagem \\ sobre a presença ou ausência \\ de dor, ocorreu em 94,8\% \\ dos prontuários analisados. \\ Ocorreu em $50 \%$ dos casos \\ no período da manhã, em \\ $79 \%$ à tarde e em $89 \%$ à \\ noite. A caracterização da dor \\ restringiu-se à descrição do \\ local $(71,1 \%)$ e da \\ intensidade (44,7\%). \\ Satisfação com analgesia foi \\ relatada por $68,4 \%$ dos \\ doentes. Cerca de 33\% dos \\ doentes relataram algum \\ grau de insatisfação com \\ analgesia.
}

\section{PALAVRAS-CHAVE}

Registros de enfermagem. Dor. Oncologia.

\author{
ABSTRACT \\ This study analyzed nursing \\ records on pain and analgesia \\ of patients admitted at a \\ cancer treatment hospital, \\ between October and \\ November of 1999, and \\ compared then to the patients \\ reports. Thirty-eight patients \\ that referred pain were \\ interviewed and had their \\ medical charts analyzed. \\ Presence or lack of pain was \\ documented in $94.8 \%$ of the \\ charts. The distribution of this \\ type of record throughout the \\ day was: $50 \%$ in the morning, \\ $79 \%$ in the afternoon, and \\ 89\% at night. Pain assessment \\ was restricted to intensity \\ (44.7\%) and location (71.1\%). \\ Sixty-eight percent of patients \\ were satisfied with the \\ analgesia provided whereas \\ about 33\% mentioned some \\ level of dissatisfaction.
}

\section{KEYWORDS}

Nursing records. Pain. Oncology.

\begin{abstract}
RESUMEN
Estudio que analizó los registros de enfermería sobre el dolor y analgesia en pacientes internados en un hospital oncológico, de octubre a noviembre de 1999. Fueron entrevistados 38 pacientes con queja dolorosa. El registro de enfermería sobre la presencia o ausencia de dolor, ocurrió en el 94,8\% de las historias clínicas analizadas. Fue identificado el $50 \%$ de los casos durante el período de la mañana, el 79\% por la tarde y el $89 \%$ por la noche. La caracterización del dolor se restringió a la descripción del local $(71,1 \%)$ $y$ de la intensidad (44,7\%). La satisfacción con analgesia fue relatada por $68,4 \%$ de los pacientes y cerca de un tercio de los pacientes relataron algún grado de insatisfacción con analgesia.
\end{abstract}

PALABRAS-CLAVE

Registros de enfermería. Dolor. Oncología.

\author{
* Dissertação de \\ mestrado apresentada \\ à Escola de \\ Enfermagem da \\ Universidade de São \\ Paulo, 2001 \\ 1 Enfermeira. Mestre \\ em Enfermagem pela \\ Escola de Enfermagem \\ da Universidade de \\ São Paulo, \\ yaraboaventura@zipmail.com.br \\ 2 Enfermeira. Professora \\ Livre-Docente do \\ Departamento de \\ Enfermagem Médico- \\ Cirúrgica da Escola \\ de Enfermagem \\ da Universidade \\ de São Paulo, \\ parpca@usp.br
}




\section{INTRODUÇÃO}

Dor é freqüente em neoplasias malignas em todas as fases e se acentua com a evolução da doença ${ }^{(1)}$. Pode estar presente em 20\% a $50 \%$ dos doentes no início do tratamento e ocorrer em cerca de 70\% - 90\% daqueles com doença avançada $^{(2)}$.

Os estudos evidenciam que a dor, quando não aliviada, limita o indivíduo nas atividades de vida diária, altera o apetite, o padrão de sono, a deambulação, a movimentação, o humor, o lazer, as atividades profissionais, sociais e familiares. Nos doentes com câncer a dor pode desencadear frustração, processo depressivo, isolamento social e familiar, exacerbação do medo e da dor. A dor, quando não tratada adequadamente, afeta a qualidade de vida dos doentes e de seus cuidadores em todas as dimensões: física, psicológica, social e espiritual ${ }^{(3-4)}$.

Apesar das evidências do impacto da dor nos doentes, ela é subidentificada e subtratada. As possíveis causas do insuficiente alívio da dor são a inadequação dos modelos de avaliação da dor e as falhas na formação dos profissionais de saúde sobre dor e analgesia, resultando no uso incorreto de terapias analgésicas ${ }^{(5-20)}$.

Dor é freqüente, cotidiana e seu controle deve ser uma preocupação do enfermeiro. A atuação do profissional, de modo independente e colaborativo, compreende a identificação de queixa álgica e a seleção de estratégias para seu controle $\mathrm{e}^{(2 ; 2)}$. A experiência dolorosa é um fenômeno individual e, para caracterizá-la, devem ser realizadas avaliações sistemáticas $^{(2 ; 21-22)}$. O registro de tais informações permite que os dados sejam compartilhados entre os diversos plantões e a equipe multiprofissional, possibilitando melhor assistência. A comunicação entre o doente e os profissionais que o atendem é de extrema importância para compreensão do quadro álgico e de seu alívio. Esse trabalho visa a analisar os registros sobre dor e analgesia.

\section{OBJETIVOS}

- Analisar os registros de enfermagem

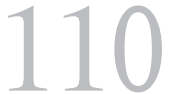

Rev Esc Enferm USP 2003; 37(2): 109-18.
- Comparar os registros de enfermagem com o relato do doente.

\section{METODOLOGIA}

Trata-se de estudo transversal do tipo descritivo-exploratório. Os dados foram coletados no período de 25 de outubro a 25 de novembro de 1999, em duas unidades médico-cirúrgicas do Centro de Tratamento e Pesquisa do Hospital do Câncer A. C. Camargo, após a aprovação pelo Comitê de Ética da referida instituição.

Foram elegíveis para o estudo os doentes hospitalizados em duas unidades médico-cirúrgicas do referido hospital com queixa de dor nas últimas 24 horas, com idade superior a 18 anos, com capacidade de compreensão e comunicação adequadas para participar da entrevista, que estavam hospitalizados há pelo menos 24 horas e que concordaram em participar do estudo e assinaram o termo de consentimento pós-informado.

A amostra foi composta por 38 doentes. Os doentes foram entrevistados uma única vez, mesmo aqueles que permaneceram internados por mais de 24 horas. Tal decisão deveu-se à possibilidade de se influir na vivência de dor do doente e de se introduzir viés no estudo.

Utilizou-se instrumento de coleta de dados composto por dados de identificação do doente (nome, registro hospitalar, sexo, idade, escolaridade, profissão e dia analisado) e da doença (tumor primário, metástases, data de internação, motivo da internação). Esses dados foram coletados no prontuário do doente. As avaliações da dor e da satisfação com analgesia, realizadas por meio de entrevista, incluíram dados sobre a caracterização da dor (local, intensidade, qualidade, duração), a identificação das atividades prejudicadas pela dor e a satisfação com a analgesia experimentada.

A análise dos registros de enfermagem no prontuário correspondeu às ultimas vinte e quatro horas, e compreendeu os mesmos itens verificados junto ao doente que referiu dor. Foram analisados os registros nos três turnos de trabalho (manhã, tarde e noite) na evolução, prescrição e anotação de enfermagem. A análise dos registros foi realizada de acordo com critérios previamente estabelecidos. 
Foi realizado teste piloto com 6 doentes internados em unidades do hospital, que não as do presente estudo, para verificar a adequação dos instrumentos. Após o teste, foi feita a revisão do instrumento de coleta, refinando sua objetividade e clareza.

Os dados estão apresentados em forma de tabelas. As freqüências foram calculadas em números absolutos e em porcentagem.
Foram realizados testes baseados na distribuição normal e de qui-quadrado ${ }^{(23)}$.

\section{RESULTADOS}

Os dados sóciodemográficos dos doentes e de características da doença e dos doentes desse estudo estão apresentados na Tabela 1 .

Tabela 1 - Distribuição da caracterização dos doentes e da doença. São Paulo, 1999.

\begin{tabular}{|c|c|c|}
\hline Variável Sóciodemográfica e da Doença & $\mathbf{n}=38$ & $\%$ \\
\hline \multicolumn{3}{|l|}{ SEXO } \\
\hline Masculino & 15 & 39,5 \\
\hline Feminino & 13 & 60,5 \\
\hline \multicolumn{3}{|l|}{ ESCOLARIDADE } \\
\hline $0|-| 4$ anos & 16 & 42,2 \\
\hline $5|-| 11$ anos & 11 & 28,9 \\
\hline $12|-| 16$ anos & 11 & 28,9 \\
\hline \multicolumn{3}{|l|}{ IDADE } \\
\hline $20|-| 39$ anos & 8 & 21,1 \\
\hline $40|-| 59$ anos & 22 & 57,8 \\
\hline $60|-| 80$ anos & 8 & 21,1 \\
\hline \multicolumn{3}{|l|}{ ATIVIDADE OCUPACIONAL REMUNERADA } \\
\hline $\operatorname{Sim}$ & 20 & 52,6 \\
\hline Não & 14 & 36,9 \\
\hline Aposentado & 4 & 10,5 \\
\hline \multicolumn{3}{|l|}{ MOTIVO DE INTERNAÇÃO } \\
\hline Cirurgia & 25 & 65,8 \\
\hline Suporte clínico & 7 & 18,5 \\
\hline Radioterapia & 1 & 2,6 \\
\hline Quimioterapia & 1 & 2,6 \\
\hline Investigação diagnóstica & 4 & 10,5 \\
\hline \multicolumn{3}{|l|}{ LOCALIZAÇÃO DO TUMOR } \\
\hline Pélvis & 12 & 31,5 \\
\hline Cabeça e pescoço & 6 & 15,8 \\
\hline Mama & 6 & 15,8 \\
\hline Sistema nervoso & 2 & 5,3 \\
\hline Abdômen & 2 & 5,3 \\
\hline Hematológico & 2 & 5,3 \\
\hline Pele & 2 & 5,3 \\
\hline Outros & 2 & 5,3 \\
\hline Desconhecido & 1 & 2,6 \\
\hline Sem neoplasia & 3 & 7,8 \\
\hline
\end{tabular}

A maioria dos doentes $(60,5 \%)$ pertenceu ao sexo feminino, a média de idade foi de 50,1 anos $(\mathrm{DP}=13,9)$, com mediana de 49,5 anos. A faixa etária predominante foi a de 40 a 59 anos (57,8\%). A média de escolaridade dos doentes foi de 9,5 anos ( $\mathrm{DP}=5,13)$, com me- diana de 11 anos. A maioria $(65,8 \%)$ estava hospitalizada para realização de cirurgia e $7,8 \%(\mathrm{n}=3)$ dos doentes não tinham neoplasia. A neoplasia pélvica foi a mais freqüente $(31,5 \%)$, seguida das localizações cabeça e pescoço e mama $(15,8 \%)$.
Análise dos registros de enfermagem sobre dor e analgesia em doentes hospitalizados 
Yara Boaventura da Silva Cibele Andrucioli M.Pimenta
Considerando-se os 38 prontuários dos doentes, observam-se anotações de presença de dor em 71,1\% $(n=27)$ dos registros de enfermagem, ausência de dor em 23,7\% (n=9) e não havia registro de presença ou ausência de dor em $5,2 \%(n=2)$.

Os registros de enfermagem também foram analisados, considerando-se os plantões da manhã, da tarde e da noite, e totalizaram 114 observações (Tabela 2).

O registro da presença ou ausência de dor ocorreu em $72 \%$ das anotações de enfermagem $(\mathrm{n}=82)$ sobre os doentes que compuseram a amostra. O registro sobre dor foi mais freqüente nos plantões tarde e noite $(79 \%$ e $89,5 \%$, respectivamente) que no plantão manhã $(47,4 \%)$.

Quando havia registro de dor em alguns dos plantões e, em outro plantão, registro de ausência de dor ou falta de registro, considerou-se o registro de presença de dor. Tal decisão deveuse ao fato de que não foi investigado com o doente o período em que ele teve dor, o que impossibilitou comparar o período do registro de enfermagem com o período em que a dor ocorreu.

Observou-se no prontuário de 9 doentes $(23,7 \%)$ registro de enfermagem constando ausência de dor nas últimas vinte e quatro horas. No entanto, todos os doentes relataram dor nas últimas vinte e quatro horas.
$\mathrm{Na}$ Tabela 3 são apresentados os dados relativos à caracterização da dor encontrados nos registros de enfermagem e no relato dos doentes.

Pela Tabela 3 nota-se que o registro de enfermagem das características da dor dos doentes que compuseram a amostra consistiu, principalmente, na descrição do local da dor em $71,1 \%(n=27)$ dos casos, e da intensidade em 44,7\% $(n=17)$ das vezes. Os doentes caracterizaram plenamente sua dor, excetuando-se um que não descreveu a qualidade de sua dor e três que não discriminaram os prejuízos dela advindos.

Dor intensa representou 58,8\% $(\mathrm{n}=10)$ dos registros, dor leve representou 41,2\% $(n=17)$ e não houve registro de enfermagem de dor moderada. Os doentes relataram dor intensa em 57,9\% $(\mathrm{n}=22)$ das vezes, dor leve em $23,7 \%(n=9)$ dos casos e $18,4 \%$ dos doentes relataram dor moderada.

Havia registro de enfermagem sobre a duração da dor de 5 doentes. Desses, 80\% $(n=4)$ referiram dor contínua e $20 \%(n=1)$ dor intermitente. Todos os doentes (100\%) descreveram a duração da dor. Registro de enfermagem sobre a qualidade da dor dos doentes ocorreu apenas em 3 prontuários.

Tabela 2 - Distribuição da existência de registro de enfermagem sobre dor, nas últimas vinte e quatro horas, nos plantões da manhã, tarde e noite dos doentes. São Paulo, 1999.

\begin{tabular}{lcc|ccccccc}
\hline Existência de Registro & \multicolumn{2}{c}{ Manhã } & \multicolumn{2}{c}{ Tarde } & \multicolumn{2}{c}{ Noite } & \multicolumn{2}{c}{ Total } \\
\hline & $\mathbf{n}^{\mathbf{0}}$ & $\mathbf{\%}$ & $\mathbf{n}^{\mathbf{0}}$ & $\mathbf{\%}$ & $\mathbf{n}^{\mathbf{0}}$ & $\mathbf{\%}$ & $\mathbf{n}^{\mathbf{o}}$ & $\mathbf{\%}$ \\
Sim & 18 & 47,4 & 30 & 79,0 & 34 & 89,5 & 82 & 72,0 \\
Não & 18 & 47,4 & 7 & 18,4 & 4 & 10,5 & 29 & 25,4 \\
Não se aplica * & 2 & 5,2 & 1 & 2,6 & 0 & 0 & 3 & 2,6 \\
Total & 38 & 100 & 38 & 100 & 38 & 100 & 114 & 100 \\
\hline
\end{tabular}

* Situações em que o doente estava fora da unidade em um dos plantões analisados.

Tabela 3 - Descrição das características da dor e dos prejuízos dela advindos existentes nos prontuários e das características relatadas pelos doentes. São Paulo, 1999.

\begin{tabular}{lcccc}
\hline \multicolumn{1}{c}{ Características da Dor } & \multicolumn{2}{c}{ Registro } & \multicolumn{2}{c}{ Doentes } \\
\hline & $\mathbf{n}^{\mathbf{o}}$ & $\mathbf{\%}$ & $\mathbf{n}^{\mathbf{o}}$ & $\%$ \\
Local & 27 & 71,1 & 38 & 100 \\
Intensidade & 17 & 44,7 & 38 & 100 \\
$\quad$ Leve & 7 & 41,2 & 9 & 23,7 \\
$\quad$ Moderada & 0 & 0 & 7 & 18,4 \\
$\quad$ Intensa & 10 & 58,8 & 22 & 57,9 \\
Duração do episódio & 5 & 13,2 & 38 & 100 \\
Qualidade & 3 & 7,9 & 37 & 97,4 \\
Prejuízos advindos da dor & 5 & 13,2 & 35 & 92,1 \\
\hline
\end{tabular}


Os prejuízos advindos da dor observados nos prontuários e os relatados pelos doentes estão apresentados na Tabela 4.

Entre os 38 doentes entrevistados, 35 referiram prejuízos relacionados à dor. Os prejuízos advindos da dor relatados pelos doentes foram os seguintes: dificuldade para movimentar-se no leito $(71,4 \%)$, alteração do sono $(60 \%)$, alteração do humor $(54,3 \%)$, dificuldade na deambulação, alteração no apetite e dificuldade para respirar e tossir foram referidas por parcela entre $30 \%$ e $50 \%$ dos doentes. A média de prejuízos relatada foi de 3,09 prejuízos por doente.

Só se encontrou 05 registros de enfermagem sobre os prejuízos advindos da dor e, em 4 vezes referiam-se à dificuldade para movimentar-se no leito.

Tabela 4 - Prejuízos advindos da dor existentes nos prontuários e os relatados pelos doentes. São Paulo, 1999.

\begin{tabular}{|c|c|c|c|c|}
\hline \multirow[t]{2}{*}{ Prejuízos } & \multicolumn{2}{|c|}{ Registro } & \multicolumn{2}{|c|}{ Doentes } \\
\hline & $\mathbf{n}^{\mathbf{o}}$ & $\%$ & $\mathbf{n}^{\mathbf{o}}$ & $\%$ \\
\hline Dificuldade para movimentar-se no leito & 4 & 80 & 25 & 71,4 \\
\hline Alteração do sono & & & 21 & 60,0 \\
\hline Alteração do humor & & & 19 & 54,3 \\
\hline Dificuldade na deambulação & & & 18 & 51,4 \\
\hline Alteração do apetite & & & 13 & 37,1 \\
\hline Dificuldade para respirar/tossir & 1 & 20 & 12 & 34,3 \\
\hline
\end{tabular}

A Tabela 5 apresenta a coincidência entre o registro de enfermagem e o relato do doente quanto a localização, intensidade, duração e qualidade da dor e do prejuízo dela advindo. Foram utilizados, para essa comparação, somente os prontuários em que havia descrição dessas características.
Pela Tabela 5 nota-se pequena coincidência entre o registro de enfermagem e o relato dos doentes quanto ao local, intensidade de dor e os prejuízos advindos da dor.

Tabela 5 - Distribuição da coincidência da caracterização da dor entre o registro de enfermagem e o relato do doente. São Paulo, 1999.

\begin{tabular}{lcccccc}
\hline \multicolumn{1}{c}{ Características da Dor } & \multicolumn{2}{c}{ Coincide } & \multicolumn{2}{c}{ Não Coincide } & \multicolumn{2}{c}{ Total } \\
\hline & $\mathbf{n}^{\mathbf{o}}$ & $\%$ & $\mathbf{n}^{\mathbf{o}}$ & $\%$ & $\mathbf{n}^{\mathbf{o}}$ & $\%$ \\
Local & 7 & 25,9 & 20 & 74,1 & 27 & 100 \\
Intensidade & 7 & 41,2 & 10 & 58,8 & 17 & 100 \\
Duração & 5 & 100 & 0 & 0 & 5 & 100 \\
Qualidade & 3 & 100 & 0 & 0 & 3 & 100 \\
Prejuízos & 1 & 20 & 4 & 80 & 5 & 100 \\
\hline
\end{tabular}

Os dados relativos à satisfação expressa pelos doentes com analgesia experimentada e à prontidão da equipe no atendimento da queixa dolorosa estão apresentados na Tabela 6.

Analisando-se a Tabela 6 nota-se que $68,4 \%$ dos doentes disseram-se satisfeitos com a analgesia obtida e $31,6 \%(n=12)$ consideraram-se parcialmente satisfeitos ou insatisfeitos. A maior parte $(65,5 \%)$ informou ter sido atendido em menos de dez minutos e todos os doentes que solicitaram medicações para o alívio da dor foram atendidos. Nove doentes não solicitaram alívio para dor.
Análise dos registros de enfermagem sobre dor e analgesia em doentes hospitalizados 
Yara Boaventura da Silva Cibele Andrucioli M.Pimenta

Tabela 6 - Distribuição do relato dos doentes sobre a satisfação com analgesia e o tempo de espera para o atendimento após a solicitação de um analgésico. São Paulo, 1999.

\begin{tabular}{lcc}
\hline Variáveis & & \\
\hline Satisfação com Analgesia & $\mathbf{n}=\mathbf{3 8}$ & $\mathbf{\%}$ \\
Satisfeito & 26 & 68,4 \\
Parcialmente satisfeito & 10 & 26,3 \\
Insatisfeito & 2 & 5,3 \\
Atendido quando Solicitado & $\mathbf{n}=\mathbf{2 9}$ & \\
Sim, solicitou analgésico e foi atendido & 29 & 100 \\
Tempo de Atendimento & $\mathbf{n}=\mathbf{2 9}$ & \\
Menos de 10' & 19 & 65,5 \\
Entre 10'e 30' & 8 & 27,6 \\
Mais de 30' & 2 & 6,9 \\
\hline
\end{tabular}

A Tabela 7 apresenta a comparação entre a satisfação e a intensidade de dor relatada pelos doentes.

Para a análise estatística da Tabela 7 agruparam-se as categorias "insatisfeito" e "parcialmente insatisfeito" da variável satisfação, e as categorias "leve" e "moderada" da variável intensidade de dor. Esses agrupamen- tos foram realizados para permitir a aplicação do teste qui-quadrado. Observou-se $\mathrm{p}=0,0501$, isto é, a satisfação e a intensidade de dor não estavam associadas. No entanto, esta afirmação deva ser tomada com cautela, uma vez que o nível descritivo observado $(\mathrm{p}=0,0501)$ foi muito próximo do nível de significância adotado $(0,05)$.

Tabela 7 - Distribuição da satisfação com analgesia recebida comparada à intensidade de dor relatada pelos doentes. São Paulo, 1999.

\begin{tabular}{lcccccccc}
\hline \multicolumn{1}{c}{$\begin{array}{c}\text { Satisfação com } \\
\text { analgesia }\end{array}$} & \multicolumn{7}{c}{ Intensidade de dor } \\
& \multicolumn{2}{c}{ Leve } & Moderada & Intensa & Total \\
& $\mathbf{n}^{\mathbf{0}}$ & $\mathbf{\%}$ & $\mathbf{n}$ & $\mathbf{0}$ & $\mathbf{n}^{\mathbf{o}}$ & $\mathbf{\%}$ & $\mathbf{n}^{\mathbf{o}}$ & $\mathbf{\%}$ \\
& 0 & 0 & 0 & 0 & 2 & 5,3 & 2 & 5,3 \\
Insatisfeito & 0 & 0 & 2 & 5,3 & 8 & 21 & 10 & 26,3 \\
Parcialmente satisfeito & 8 & 21 & 5 & 13,2 & 13 & 34,2 & 26 & 68,4 \\
Satisfeito & $\mathbf{8}$ & $\mathbf{2 1}$ & $\mathbf{7}$ & $\mathbf{1 8 , 5}$ & $\mathbf{2 3}$ & $\mathbf{6 0 , 5}$ & $\mathbf{3 8}$ & $\mathbf{1 0 0}$ \\
Total & & & & & & & &
\end{tabular}

\section{DISCUSSÃO}

A Joint Commission on Accreditation of Healthcare Organization (JCAHO) ${ }^{(24-25)}$, entidade norte-americana de avaliação de hospitais incluiu o alívio da dor como item a ser avaliado na acreditação hospitalar, a partir de 2001. Tal decisão resultou no reconhecimento que hoje se tem sobre o direito de o doente ter sua queixa dolorosa adequadamente avaliada, registrada e controlada. A JCAHO estabelece padrões para o manejo da dor em serviços ambulatoriais, domiciliares, de saúde mental, de reabilitação e em hospitais ${ }^{(24-25)}$.
A existência de queixa dolorosa deve ser investigada durante toda a internação. A avaliação da dor inclui a caracterização do local, da intensidade, da freqüência, da duração e da qualidade do sintoma, e deve ser registrada em instrumentos desenvolvidos pela instituição. A JCAHO verifica a implementação desses padrões analisando a existência de registro em prontuário desses itens, entre outras estratégias $^{(25)}$. Ainda segundo a recomendação da $\mathrm{JCAHO}^{(25)}$, cabe à organização hospitalar buscar informações sobre seu desempenho no alívio da dor, por meio de indicadores de qualidade, como a verificação das 
necessidades, das expectativas e da satisfação de doentes, família e profissionais. Tais recomendações vêm ao encontro das premissas deste trabalho.

No presente estudo, construiu-se um instrumento para se analisar o registro de enfermagem sobre dor que se assemelha ao proposto pela JOINT. Assim, esta análise compreendeu a presença de dor, a localização, a intensidade, a qualidade, a duração, os prejuízos para as atividades de vida diária e o alívio obtido.

A existência de registro de enfermagem sobre dor e analgesia foi avaliada de dois modos: considerando-se o prontuário como um todo (relativo às últimas 24 horas) e discriminando-se as anotações por plantão (manhã, tarde e noite).Foram analisados 38 prontuários e 114 registros de enfermagem.

Registros de enfermagem sobre a presença ou ausência de dor foram observados em $94,8 \%(n=36)$ dos casos. A existência de, no mínimo, um registro sobre dor nas últimas vinte e quatro horas, levou-nos a considerar que havia registro sobre dor. No entanto, quando se considerou o registro por plantão $(n=114)$, observou-se que, em cerca de $1 / 4$ dos casos (Tabela 2), não havia qualquer menção à presença ou à ausência de dor. Em quase $30 \%$ dos casos, havia registro de ausência de dor, embora todos os doentes $(n=38)$ tenham sentido dor, nas últimas vinte e quatro horas.

O registro sobre as características da dor foi pobre, pois pouco havia além da descrição da localização $(71,1 \%)$ e da intensidade (44,7\%). No entanto, quando indagados pela pesquisadora, todos os doentes caracterizaram plenamente a sua dor. Nessa caracterização a dor não foi desprezível e deveria ter sido cuidada. Para aproximadamente $60 \%$ dos doentes, a dor foi descrita como intensa; para quase $20 \%$ deles, a dor foi moderada e para mais de $50 \%$, a dor foi contínua.

A dor trouxe vários prejuízos para a maioria dos doentes $(n=35)$, com média de 3,09 prejuízos por doente.

Os achados do presente estudo assemelham-se aos de outros trabalhos ${ }^{(14 ; 26-28)}$. Em estudo que analisou o registro de enfermagem e o relato de 65 doentes em pós-operatório também se verificou que a documentação sobre dor era escassa e que a experiência dolorosa relatada pelo doente não coincidiu com os registros $^{(26)}$. Em estudo que envolveu 84 doentes no pós-operatório, observou-se que registro sobre a dor ocorreu em menos de $50 \%$ das vezes, quando comparados ao relato do doente ${ }^{(27)}$. Num estudo com 34 doentes cirúrgicos, encontraram-se, em 73,5\% das vezes, registros sobre dor no pós-operatório imediato. No entanto, pouco havia de sua caracterização além de local $(50 \%)$ e intensidade (17,6\%), o que também se assemelha aos resultados do presente estudo ${ }^{(14)}$. Quando se analisaram os prontuários de 136 doentes cirúrgicos, verificou-se ausência de registro sobre dor em $77 \%$ deles. Considerou-se documentação existente quando havia registro do local, da duração ou da intensidade de dor. Em 10\% deles, havia registro de intensidade, em $12 \%$ de local e intensidade e, em 0,07\%, da duração da dor ${ }^{(28)}$. A análise dos registros de enfermagem sobre dor, realizada no presente estudo, mostrou índices superiores ao descrito anteriormente.

Na presente pesquisa, a coincidência entre o registro de enfermagem e o relato do doente foi pequena. Houve coincidência quanto ao local e a intensidade em 7 casos; a duração, em 5 vezes; a qualidade, em 3 casos e, em 1 oportunidade, quanto aos prejuízos da dor. Cabe destacar que, em 30\% das vezes, havia registro de que o doente não teve dor nas últimas vinte e quatro horas do período avaliado, o que contradiz o relato dos doentes.

Comparando-se os resultados do presente estudo com os observados por Pinheiro ${ }^{(29)}$, encontram-se semelhanças e diferenças. Pinheiro ${ }^{(29)}$ analisou a coincidência entre o relato do doente e a percepção dos profissionais sobre o local e a intensidade da dor resultante de 263 procedimentos dolorosos. O estudo foi realizado com 50 doentes em pósoperatório de revascularização do miocárdio. Ao se comparar a opinião dos doentes com a dos profissionais sobre a intensidade da dor, observou-se por parte dos profissionais, subestimação da dor oriunda da aspiração endotraqueal, da retirada do dreno pleural e do dreno de mediastino, e superestimação da dor advinda do exercício respiratório ${ }^{34}$. No presente estudo, a coincidência de local e intensidade foi inferior à observada por Pinheiro. Possivelmente, isso esteja relacionado com o fato de que, no estudo sobre procedimentos dolorosos, o local da dor fica mais facilmente evidenciado.
Análise dos registros de enfermagem sobre dor $\mathrm{e}$ analgesia em doentes hospitalizados 
Yara Boaventura da Silva Cibele Andrucioli M.Pimenta

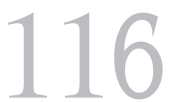

Rev Esc Enferm USP 2003; 37(2): 109-18.
A investigação da satisfação com a analgesia é parte dos protocolos de dor e analgesia e é considerada como um indicador de qualidade pela American Pain Society ${ }^{(30-33)}$. Estudos evidenciam que o doente hospitalizado com dor não aliviada tende a avaliar negativamente todos os demais serviços durante sua internaçãa ${ }^{(31)}$. Os aspectos que devem ser investigados são a satisfação do doente com a informação recebida na internação, a expectativa de dor anterior ao tratamento, a experiência dolorosa posterior, comparandoa com a expectativa, a satisfação pelo modo como enfermeiros e médicos trataram sua queixa dolorosa, o alívio obtido após intervenções analgésicas e o tempo decorrido para a realização destas ${ }^{(31-34)}$.

No presente estudo, $68,4 \%$ dos doentes disseram-se satisfeitos com a analgesia. Embora a maioria dos doentes $(68,4 \%)$ considerou-se satisfeita com a analgesia obtida, 76,3\% deles referiram dor de moderada à intensa. Na presente pesquisa, aproximadamente metade dos doentes definiu satisfação com analgesia como "total ausência de dor", outros definiram como "saber que há medicação analgésica prescrita", "perceber que a equipe está preocupada em aliviar a dor, mesmo que não tenham obtido alívio completo". Essas opiniões coincidem com as observadas em outros estudos ${ }^{(31 ; 33)}$. $\mathrm{Na}$ presente pesquisa, cerca de $1 / 3$ dos doentes relatou algum grau de insatisfação com analgesia. Esse número é significativo, considerando-se que os doentes estavam internados, a pesquisadora era profissional da instituição, usando uniforme e crachá, o que pode ter inibido a expressão mais livre de opiniões pelos doentes. A maior parte desses doentes tinha dor intensa. Contrapõe-se aos resultados anteriores o estudo de Martins ${ }^{(35)}$ com 110 doentes cirúrgicos. Observou-se significativa parcela de insatisfação com analgesia, que variou de acordo com o porte da cirurgia. Encontrou-se 67,4\% de insatisfação com a analgesia nas cirurgias de grande porte, $30 \%$ nas cirurgias de médio porte e $15,4 \%$ nas cirurgias de pequeno porte ${ }^{(35)}$.

Não houve relação estatisticamente significante entre a satisfação e a intensidade de dor, embora o p observado tenha sido limítrofe $(p=0,0501)$. Em estudo ${ }^{(36)}$ realizado em 1998, na mesma instituição, os doentes mostraram-se satisfeitos com analgesia em
$74,4 \%$ dos casos. Também nesse estudo, a freqüência e intensidade da queixa dolorosa foram significativas e contrariamente os doentes mostraram-se satisfeitos.

Em estudo sobre a percepção dos doentes sobre o alívio da dor, após cirurgias de histerectomia $(\mathrm{n}=51)$ e colicistectomia $(\mathrm{n}=50)$, verificou-se que a expectativa de alívio da dor era baixa e que cerca de $70 \%$ dos doentes tiveram dor de intensidade moderada a intensa. Em somente $50 \%$ das vezes, os profissionais investigaram o alívio da dor ${ }^{(11)}$.

No presente estudo, para $65,5 \%$ dos doentes $(n=19)$, o tempo estimado de atendimento, para o alívio da dor, foi menor do que dez minutos. Em estudo ${ }^{(33)}$ em que se avaliou o tempo para a administração de analgésico, o intervalo de até quinze minutos para ser medicado ocorreu em $60 \%$ dos doentes $(n=217)$ adultos e em $22 \%$ de crianças $(n=31)$, o que se assemelha aos dados da presente pesquisa.

No que diz respeito ao registro, enfoque dessa pesquisa, os trabalhos apontam que esse aspecto é problemático ${ }^{(37-39)}$, mesmo quando da utilização de instrumentos de avaliação padronizados ${ }^{(40-41)}$. No entanto, registros são forma de comunicação entre as equipes e entre os turnos, além de fonte de pesquisa e base para a auditoria. Sua inadequação compromete a assistência ao doente e seu aperfeiçoamento é meta a ser perseguida.

$\mathrm{Na}$ análise da evolução e prescrição de enfermagem, atividades realizadas privativamente pelo enfermeiro, percebeu-se que havia pouca referência sobre dor e sua caracterização nas evoluções diárias, mesmo para os doentes hospitalizados unicamente para o controle álgico. Em geral, a prescrição de enfermagem foi vaga no aspecto controle álgico, constando apenas de "observar e comunicar queixas álgicas", o que provavelmente deve repercutir na anotação da equipe de enfermagem e na assistência ao doente com dor.

\section{CONCLUSÕES}

Registro sobre presença ou ausência de dor nas últimas vinte e quatro horas ocorreu em $94,8 \%$ dos prontuários. Registros de enfermagem sobre as características da dor restringiram-se à descrição do local $(71,1 \%) \mathrm{e}$ da intensidade (44,7\%). Em pequeno número 
de casos houve registro da duração $(13,2 \%)$ e qualidade $(7,9 \%)$ da dor. Encontrou-se que $68,4 \%$ dos doentes $(n=26)$ consideraram-se satisfeitos com a analgesia, contrariamente à intensidade, à duração e aos prejuízos advindos da dor relatada. Cerca de $1 / 3$ dos doentes relatou algum grau de insatisfação com a analgesia.

Na comparação dos registros de enfermagem sobre dor e analgesia com o relato dos doentes observou-se pequena coincidência. $\mathrm{O}$ registro sobre as características da dor foi po- bre, embora todos os doentes, quando indagados, tenham descrito seu quadro álgico quanto ao local, intensidade, duração, qualidade e prejuízos advindos da dor. Coincidência entre o registro e o relato ocorreu em 7 casos, quanto ao local e intensidade; em 5 vezes, quanto à duração e aos prejuízos dela advindos e, em 3 oportunidades, sobre a qualidade da dor.

Acredita-se que a adoção de um padrão de avaliação diária do doente, especificamente sobre dor, possa contribuir para o aperfeiçoamento da assistência de enfermagem.

\section{REFERÊNCIAS}

(1) Bonica JJ. Treatment of cancer pain current status and future needs. In: Fields HL, Dubner $\mathrm{R}$, Cervero F. Advances in pain research and therapy: proceeding of the fourth world congress of pain, New York, Raven v. 9, p. 589-615, 1985.

(2) Pimenta CAM, Teixeira MJ. Avaliação da dor. Rev Med São Paulo 1997; 76(1):27-35.

(3) Ferrell BR The impact of pain on quality of life. A decade of research. Nurs Clin North Am 1995; 30(4):609-24.

(4) Ferrell BR, Wisdom C, Rhiner M, Aletto J. Pain management as a quality of care outcome. J Nurs Qual Assur 1991; 5(2):50-8.

(5) Clarke E, French B, Bilodeau, Capasso V.C, Edwards A, Empoliti J. Pain management knowledge, attitudes and clinical practice: the impact of nurses' characteristics and education. J Pain Symptom Manage 1996; 11(1):18-31.

(6) Ferrell BR, Cohen MZ, Rhiner M, Rozek A. Pain as a metaphor for illness. part ii: family caregivers'management of pain. Oncol Nurs Forum 1991; 18(8):1315-21.

(7) Ferrell BR, Eberts MT, Mccaffery M, Gran M. Clinical decision making and pain. Cancer Nurs 1991; 14(6):289-91.

(8) Ferrell BR, Ferrell BA, Rhiner M, Grant M. Family factors influencing cancer pain management. Postgrad Med J 1991; 67 (Suppl. 2):S64-S69.

(9) Ferrell BR, Rhiner M, Cohen MZ, Grant M. Pain as a metaphor for illness. Part I: impact of cancer pain on family caregivers. Oncol Nurs Forum 1991; 18(8):1303-09.

(10) Gordon DB, Ward SE. Correcting patient misconceptions about pain. AJN 1995; 95(7):43-5.
(11) Kuhn S, Cooke K, Collins M, Jones J.M, Mucklow JC. Perceptions of pain relief after surgery. BMJ 1990; 300(30):1687-90.

(12) Marks RM, Sachar, EJ. Undertreatment of medical inpatients with narcotic analgesics. Ann Int Med 1973; 78:173-81.

(13) Melzack R, Abbott FV, Zackon W, Mulder DS, Davis MWL. Pain on a surgical ward: a survey of the duration and intensity of pain and the effectiveness of medication. Pain 1987; 29(1):67-72.

(14) Paice JA, Toy C, Shott S. Barriers to cancer pain relief: fear of tolerance and addiction. J Pain Sympton Manage 1998; 16(1):1-9.

(15) Pimenta CAM, Koizumi MS. Analgesia em câncer: crenças e atualização. Rev Esc Enferm USP 1993; 27(2):309-14.

(16) Pritchard AP. Management of pain and nursing attitudes. Cancer Nurs 1998; 11(3):203-9.

(17) Riddell A, Fitch M. Patients'knowledge of and attitudes toward the management of cancer pain. Oncol Nurs Forum 1997; 24(10):1775-1784.

(18) Ryan P, Vortherms R, Ward S. Cancer pain: knowledge, attitudes of pharmacologic management. J Gerontol Nurs 1994; 20(1): $7-16$.

(19) Sriwatanakul K, Weis O.F, Alloza JL, Kelvie W, Weintraub M, Lasagna L. Analysis of narcotic analgesic usage in the treatment of postoperative pain. JAMA 1983; 250(7):926-9.

(20) Teixeira MJ, Shibata MK, Pimenta CAM, Corrêa CF. Dor no Brasil: estado atual e perspectivas. São Paulo: Limay; 1995.
Análise dos registros de enfermagem sobre dor $\mathrm{e}$ analgesia em doentes hospitalizados 
Yara Boaventura da Silva Cibele Andrucioli M.Pimenta
Recebido: $02 / 04 / 2002$ Aprovado: 20/05/2003

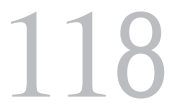

Rev Esc Enferm USP 2003; 37(2): 109-18.
(21) Melzack R, Katz J. Pain measurement in persons in pain. In: Melzack R. Textbook of pain. $3^{\text {nd }}$ ed. Edinburgh: Churchill Livingstone; 1994. p.337-51.

(22) Pimenta CAM, Cruz DALM, Santos JLF. Instrumentos para avaliação da dor. o que há de novo em nosso meio. Arq Bras Neurocir 1998; 17(1):15-24.

(23) Morettin PA, Bussab WO. Estatística básica. $4^{a}$ ed. São Paulo: Atual; 1987

(24) Dahl JL. New JCAHO standards focus on pain management. Oncology issues [serial online] 1999; 14(5):27-28. Available from: $<$ http://www.medscape.com> (16 dec. 2000)

(25) Joint Commission on Acrreditation of Healthcare Organization. [online]. Comprehensive accreditation manual for hospitals: the official handbook (camh). Available from: $<$ http://www.jcaho.org/standard $>$ (16 dec. 2000)

(26) Briggs M, Dean KL. A qualitative analysis of the nursing documentation of post-operative pain management. J Clin Nurs 1998; $7(2): 155-63$.

(27) Camp LD, O'Sullivan PS. Comparison of medical, surgical and oncology patient's descriptions of pain and nurse's documentation of pain assessments. J Adv Nurs 1987;12(5):593-8.

(28) Lellan KM. A chart audit rev.iewing the prescription and administration trends of analgesia and the documentation of pain, after surgery. J Adv Nurs 1997; 26:345-50.

(29) Pinheiro VFO. Dor no pós-operatório de cirurgia cardíaca. [dissertação]. São Paulo (SP): Escola de Enfermagem da USP; 2000.

(30) American Pain Society Quality of Care Committe. Quality improvement guidelines for rhe treatment of acute pain and cancer pain. JAMA 1995; 274 ( 23):1874-80.

(31) Jamison RN, Ross MJ, Hoopman P, Griffin F, Levy J, Daly M, Schaffer JL. Assessment of postoperative pain management: pain satisfaction and perceived helpefulness. Clin J Pain 1997; 13(3):229-36.

(32) Miaskowski C, Nichols R, Brody R, Synold $\mathrm{T}$. Assessment of patient satisfaction utilizing the american pain society's quality assurance standards on acute and cancer-related pain. J. Pain Sympton Manage 1994; 9(1):5-11.

(33) Ward SE, Gordon D. Application of the American Pain Society Quality Assurance Standards. Pain 1994; 56(3):299-306.
(34) Corrizzo CC, Baker MC, Henrelmann GC Assessment of patient satisfaction with pain management in community inpatient and outpatient settings. Oncol Nurs Forum 2000; 27(8):1279-86.

(35) Martins LMM, Pimenta CAM. Dor e satisfação com a analgesia no pós-operatório. In: Anais do $3^{\circ}$ Simpósio Internacional da Dor; 1997 set 19-21; São Paulo. São Paulo: Grupo de Estudos da Dor do HC/FMUSP; 1997. p. $18-9$.

(36) Santos EMM, Pimenta CAM. Contradições entre o relato de dor no pós-operatório e a satisfação do doente com a analgesia. Rev Bras Cancerol 2000; 46(1):93-104. 